\title{
Thermal Control of Spin Excitations in the Coupled Ising-Chain Material $\mathbf{R} b \mathrm{CoCl}_{3}$
}

\author{
M. Mena, ${ }^{1,2}$ N. Hänni, ${ }^{3,4}$ S. Ward ${ }^{2,5}$ E. Hirtenlechner, ${ }^{2,6}$ R. Bewley, ${ }^{7}$ C. Hubig, ${ }^{8,9}$ U. Schollwöck, ${ }^{8}$ \\ B. Normand, ${ }^{10}$ K. W. Krämer, ${ }^{3}$ D. F. McMorrow, ${ }^{1}$ and Ch. Rüegg ${ }^{5,10,11,12}$ \\ ${ }^{1}$ London Centre for Nanotechnology and Department of Physics and Astronomy, University College London, \\ Gower Street, London WC1E 6BT, United Kingdom \\ ${ }^{2}$ Laboratory for Neutron Scattering and Imaging, Paul Scherrer Institute, CH-5232 Villigen PSI, Switzerland \\ ${ }^{3}$ Department of Chemistry and Biochemistry, University of Bern, Freiestrasse 3, CH-3012 Bern, Switzerland \\ ${ }^{4}$ Physikalisches Institut, University of Bern, Siedlerstrasse 5, CH-3012 Bern, Switzerland \\ ${ }^{5}$ Department of Quantum Matter Physics, University of Geneva, CH-1211 Geneva 4, Switzerland \\ ${ }^{6}$ Institut Laue Langevin, CS 20156, F-38042 Grenoble, France \\ ${ }^{7}$ ISIS Facility, STFC Rutherford Appleton Laboratory, Harwell Campus, Didcot OX11 0QX, United Kingdom \\ ${ }^{8}$ Arnold Sommerfeld Center for Theoretical Physics, Ludwig-Maximilians-University Munich, 80333 München, Germany \\ ${ }^{9}$ Max-Planck-Institut für Quantenoptik, 85748 Garching, Germany \\ ${ }^{10}$ Paul Scherrer Institute, CH-5232 Villigen PSI, Switzerland \\ ${ }^{11}$ Institute for Quantum Electronics, ETH Zürich, CH-8093 Zürich, Switzerland \\ ${ }^{12}$ Institute of Physics, Ecole Polytechnique Federale de Lausanne, CH-1015 Lausanne, Switzerland
}

(Received 19 April 2020; accepted 27 May 2020; published 22 June 2020)

\begin{abstract}
We have used neutron spectroscopy to investigate the spin dynamics of the quantum $(S=1 / 2)$ antiferromagnetic Ising chains in $\mathrm{RbCoCl}_{3}$. The structure and magnetic interactions in this material conspire to produce two magnetic phase transitions at low temperatures, presenting an ideal opportunity for thermal control of the chain environment. The high-resolution spectra we measure of two-domain-wall excitations therefore characterize precisely both the continuum response of isolated chains and the "Zeeman-ladder" bound states of chains in three different effective staggered fields in one and the same material. We apply an extended Matsubara formalism to obtain a quantitative description of the entire dataset, Monte Carlo simulations to interpret the magnetic order, and finite-temperature density-matrix renormalization-group calculations to fit the spectral features of all three phases.
\end{abstract}

DOI: 10.1103/PhysRevLett.124.257201

Quantum systems that display one-dimensional (1D) nature [1] and Ising exchange [2] exhibit a very rich variety of phenomena. These include a gapped excitation continuum [3-5], excited bound states with emergent $E_{8}$ symmetry [6], quantum criticality [7-9], and topological excitations [10]. Recent intense interest in coupled Ising-chain physics was sparked by the ferromagnetic (FM) material $\mathrm{CoNb}_{2} \mathrm{O}_{6}$ [6,11-13], whose spectrum of magnetic excitations is divided between a kinetic bound state and a two-domainwall continuum, which itself splits into confined $\left(E_{8}\right)$ bound states. The antiferromagnetic (AFM) $A \mathrm{Co}_{2} \mathrm{~V}_{2} \mathrm{O}_{8}$ ( $A=\mathrm{Ba}, \mathrm{Sr}$ ) compounds [14-17] have spiraling Ising chains with significant Heisenberg interactions, not only separating the transverse and longitudinal bound-state excitations $[18,19]$ but also realizing both uniform and staggered, longitudinal and transverse applied fields [15] in which to measure different types of bound-state and critical behavior [10,20-22]. However, both the response of a truly decoupled Ising chain and distinguishing the temperature dependences of all these features remain as challenging problems.

A paradigm for Ising-chain physics is provided by the $\mathrm{ACoX}_{3}$ hexagonal perovskites [space group $\mathrm{Pb}_{3} / \mathrm{mmc}$, shown in Figs. 1(a) and 1(b)], which include $\mathrm{CsCoCl}_{3}$
[3,4,23-25], $\mathrm{CsCoBr}_{3}[4,5,23,24], \mathrm{RbCoCl}_{3}$ [24,26], and $\mathrm{TlCoCl}_{3}$ [27]. Their in-chain interactions are AFM and their spectra provided early examples of the continuum arising from pairs of moving domain walls (also referred to as "kinks" and "solitons"). Their special feature is that the Ising chains form a triangular lattice [Fig. 1(b)], frustrating the AFM interchain interactions. They typically show two magnetic ordering transitions, which in $\mathrm{RbCoCl}_{3}$ occur at $T_{N 1}=28 \mathrm{~K}$ and $T_{N 2}=12 \mathrm{~K}$ [28]. Thus, in contrast to $\mathrm{CoNb}_{2} \mathrm{O}_{6}$ and $A \mathrm{CO}_{2} \mathrm{~V}_{2} \mathrm{O}_{8}$, different chains experience different environments at different temperatures. Treating the neighboring chains as an effective staggered field, when this is zero one expects the spectrum of the isolated chain, a continuum with cosinusoidal boundaries [29], whereas for finite staggered fields the spectrum is a Zeeman ladder of bound states [30].

In this Letter, we study the magnetic excitations of $\mathrm{RbCoCl}_{3}$ in unprecedented detail by combining stateof-the-art instrumentation for neutron spectroscopy with a systematic thermal control of the different effectivefield contributions. This allows an unambiguous separation of isolated-chain and staggered-field physics. Rising temperature causes both a band narrowing and a broadening of 


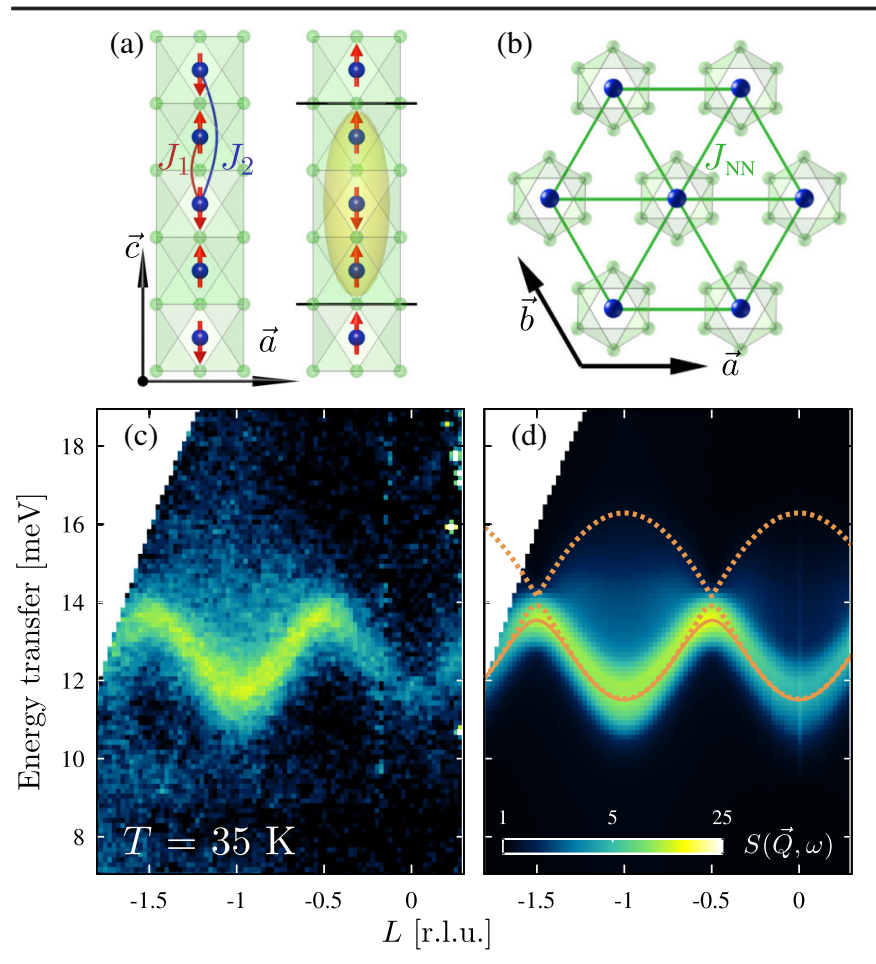

FIG. 1. (a) Schematic representation of chains in $\mathrm{RbCoCl}_{3}$. The $\mathrm{Co}^{2+}$ ions (blue) are embedded in $\mathrm{Cl}^{-}$antiprisms (light green), an environment in which their ground state is an Ising doublet. The resulting effective $S=1 / 2$ spins (red arrows) order antiferromagnetically along the $c$ axis. The yellow ellipse denotes a pair of interacting domain walls. (b) Triangular lattice of chains in $\mathrm{RbCoCl}_{3}$. (c) Scattered intensity $S(\vec{Q}, \omega)$ measured at $35 \mathrm{~K}$. (d) $S(\vec{Q}, \omega)$ calculated by combining DMRG results with extended Matsubara analysis (see text). Dotted orange lines mark the edges of the continuum; the solid orange line marks the bound mode that separates from the continuum due to the interaction $J_{2}[(\mathrm{a})]$.

bound and continuum features, as well as strong changes to the interchain coupling effects. These we explain by combining an extended Matsubara description with finite-temperature, time-dependent density-matrix renormalization-group (DMRG) calculations.

Three high-quality $\mathrm{RbCoCl}_{3}$ single crystals of total mass $7.6 \mathrm{~g}$ were grown by the Bridgman technique in a moving vertical furnace [28] and coaligned in the $(H H L)$ plane. Inelastic neutron scattering experiments were performed on the direct-geometry time-of-flight spectrometer LET (ISIS, UK) [31]. High-statistics data were collected with incident energy $E_{i}=25 \mathrm{meV}$ and $(00 L)$ perpendicular to the incident beam, at temperatures of 4,18 , and $35 \mathrm{~K}$, i.e., in each magnetically ordered phase and above both. Lowerstatistics data were collected at $8,10.5$, and $23 \mathrm{~K}$ with $E_{i}=20 \mathrm{meV}$. The data were corrected for detector efficiency and outgoing-to-incoming wave-vector ratio $k_{f} / k_{i}$ using the program MANTID [32]. Datasets for the scattered intensity $S(\vec{Q}, \omega)$ were analyzed with the HORACE software package [33].

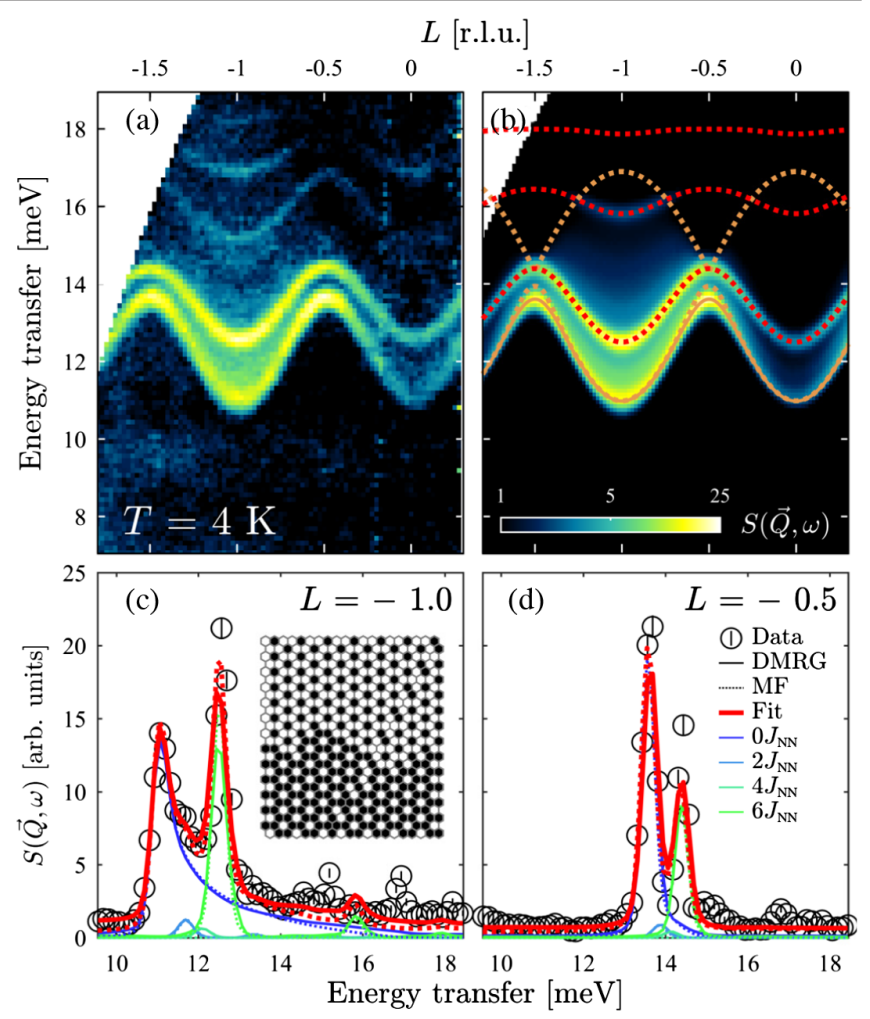

FIG. 2. (a) $S(\vec{Q}, \omega)$ measured at 4 K. (b) $S(\vec{Q}, \omega)$ calculated in Matsubara formalism (MF) with the parameters in the text. Orange lines as in Fig. 1(d). Red lines show the bound-state excitations of the Zeeman ladder. (c),(d) $S(\vec{Q}, \omega)$ at $4 \mathrm{~K}$ (black symbols) integrated over windows $-1.05<L<-0.95$ (c) and $-0.55<L<-0.45$ (d). Red lines show optimized fits obtained by summing contributions from chains in different effective fields (blue-to-green lines). These panels are enlarged in Fig. S5 of the SM [34]. The inset in (c) shows a typical planar spin configuration, dominated by FI domains, obtained from CHB Monte Carlo simulations at $T<T_{N 2}$; black hexagons denote up spins and white down spins.

We begin with an overview of the experimental data, which are shown in Figs. 1(c), 2(a), 2(c), 2(d), 3(a), 3(c), 3(d), 4(a), and 4(b). First, we identify a band of excitations in the one-spin-flip (two-domain-wall) sector with a spin gap of $11 \mathrm{meV}$ and a cosinusoidal dispersion [Fig. 1(c)]. Second, our measurements confirm the strongly 1D nature of $\mathrm{RbCoCl}_{3}$, as shown in Sec. S1 of the Supplemental Material (SM) [34]. All data presented here were therefore averaged over $H$ and $K$ and binned as functions of the energy transfer and the momentum along $L$. In detail, the width of the band we observe at $35 \mathrm{~K}$ [Fig. 1(c)] far exceeds the instrumental resolution, and its line shape [Fig. 4(a)] suggests a continuum. The spectrum sharpens at $18 \mathrm{~K}$ [Fig. 3(a)], presenting more lines with more intensity and less broadening [Figs. 3(c) and 3(d)]. At $4 \mathrm{~K}$, the scattered intensity splits clearly into two different types of feature [Fig. 2(a)], a continuum [Fig. 2(c)] whose maximum disperses between 11 and $13.5 \mathrm{meV}$ and a set of resolution-limited modes, the Zeeman ladder, whose lowest 
member [Fig. 2(d)] disperses between 12.5 and $14.5 \mathrm{meV}$. It is evident that thermal effects go beyond a simple line broadening and include effective control of the staggered field due to the different 3D ordered phases.

To illustrate the influence of magnetic order on the measured Ising-chain dynamics, we have implemented a cluster heat bath (CHB) Monte Carlo algorithm, as detailed in Sec. S2 of the SM [34]. Developed originally for studies of $A \mathrm{Co} X_{3}$ compounds [38-40], CHB simulations below $T_{N 2}$ show well-ordered chains aligning to form domains of ferrimagnetic (FI) "honeycomb" planar order [40,41], represented in the inset of Fig. 2(c). Interchain interactions are dominated by the nearest-neighbor (NN) AFM coupling $J_{\mathrm{NN}}$ [Fig. 1(b)], whose frustration leads to a massive degeneracy, and the FI order is selected by a weak FM next-nearest-neighbor (NNN) term $J_{\mathrm{NNN}}$ [41]. The partially disordered AFM (PDAFM) [25,41] state between $T_{N 2}$ and $T_{N 1}$ is characterized primarily by a decrease in FI interchain order [inset of Fig. 3(c)], while the staggered magnetization within each chain is also impacted weakly [28] by thermally excited domain walls [42]. Although 3D order is lost above $T_{N 1}$, both susceptibility and diffuse scattering measurements [28] suggest that anomalously slow shortrange correlations persist up to $60-80 \mathrm{~K}$, and we will quantify this effect.

The Hamiltonian of a single Ising-Heisenberg chain with NN and NNN in-chain interactions [Fig. 1(a)] is

$$
\begin{aligned}
\mathcal{H}= & \sum_{j} 2 J_{1}\left[S_{j}^{z} S_{j+1}^{z}+\epsilon_{1}\left(S_{j}^{x} S_{j+1}^{x}+S_{j}^{y} S_{j+1}^{y}\right)\right]+h_{j} S_{j}^{z} \\
& +2 J_{2}\left[S_{j}^{z} S_{j+2}^{z}+\epsilon_{2}\left(S_{j}^{x} S_{j+2}^{x}+S_{j}^{y} S_{j+2}^{y}\right)\right],
\end{aligned}
$$

where $\left|J_{2}\right| \ll J_{1}, \epsilon_{1} \ll 1$, and $h_{j}$ is the effective staggered field due to magnetic order. For this class of compounds, $J_{2}$ is thought to be FM [24]. An alternative approach [25] using only $\mathrm{NN}$ terms and including an anisotropic splitting of the $\mathrm{Co}^{2+}$ Kramers doublet is also thought [43] to provide a complete treatment of the orbital terms.

In this formalism, which we term the "Matsubara framework," the matrix elements of Eq. (1) expressed in terms of the separation $\nu$ of two domain walls are

$$
\begin{aligned}
\langle 1|\mathcal{H}(\vec{Q})| 1\rangle & =2 J_{1}\left(1+\epsilon_{1}^{2}\right)+2 J_{2}\left[1-\epsilon_{2} \cos (2 \pi L)\right]+h, \\
\langle\nu|\mathcal{H}(\vec{Q})| \nu\rangle & =2 J_{1}\left(1+\frac{3}{2} \epsilon_{1}^{2}\right)+4 J_{2}+\nu h, \\
\langle\nu|\mathcal{H}(\vec{Q})| \nu \pm 2\rangle & =\epsilon_{1} J_{1}\left(1+e^{\mp 2 i \pi L}\right), \\
\langle\nu|\mathcal{H}(\vec{Q})| \nu \pm 4\rangle & =-\frac{1}{2} \epsilon_{1}^{2} J_{1}\left(1+e^{\mp 4 i \pi L}\right),
\end{aligned}
$$

and $\left\langle\nu|\mathcal{H}(\vec{Q})| \nu^{\prime}\right\rangle=0$ otherwise $[24,29,43]$. As noted above, the spectrum of this Hamiltonian forms a continuum when $h=0$ [dashed orange lines in Figs. 1(d), 2(b), and 3(b)]

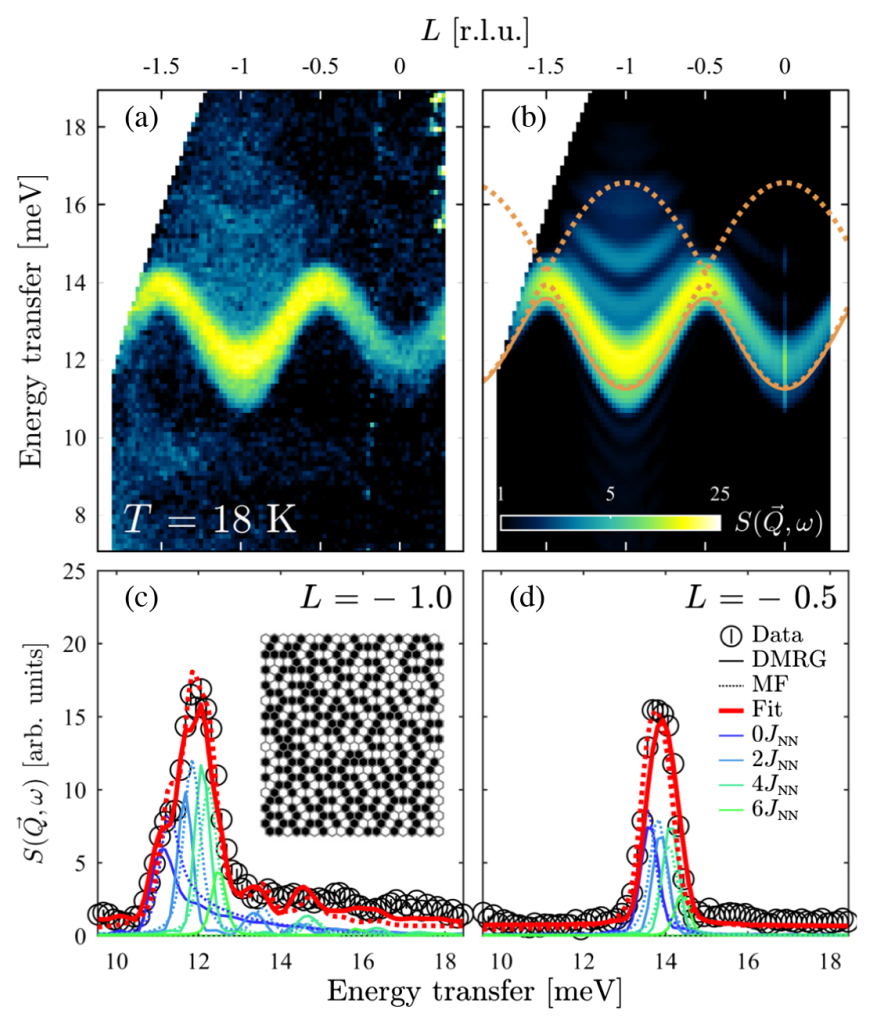

FIG. 3. (a) $S(\vec{Q}, \omega)$ measured at $18 \mathrm{~K}$. (b) $S(\vec{Q}, \omega)$ calculated by DMRG. Orange lines as in Fig. 1(d). (c),(d) Integrated scattered intensities following the conventions of Figs. 2(c) and 2(d). These panels are enlarged in Fig. S5 of the SM [34]. The inset in (c) shows a typical planar spin configuration in the PDAFM phase from CHB Monte Carlo.

and a Zeeman ladder when $h>0$. With $h=0$ but $J_{2}$ nonzero, an additional bound mode separates from the continuum around half-integer values of $L$ (solid orange lines), while the intensity in the continuum shifts towards its lower edge. By calculating the spectral weights from the Green function [29], the full dynamical structure factor shown in Fig. 2(b) was computed using Eq. (1) with the parameters discussed below.

Because $J_{\mathrm{NNN}}$ is much smaller than the instrumental resolution, at $4 \mathrm{~K}$ we model the effective staggered field by taking $h=m J_{\mathrm{NN}}$ with $m \in\{0,2,4,6\}$. For perfect FI order, $2 / 3$ of the sites experience a vanishing field, $h=0$, and $1 / 3$ a field $h=6 J_{\mathrm{NN}}$; in practice, planar domain boundaries between different FI regions [inset Fig. 2(c)] produce small numbers of chains subject to the two intermediate fields. At $18 \mathrm{~K}$, the PDAFM regime presents a mix of all four staggered fields [inset of Fig. 3(c)]. At $35 \mathrm{~K}$, more detailed considerations are required as the chain environment becomes increasingly random.

Concerning in-chain dynamics at finite temperatures, the domain-wall pair excited by the scattered neutron is itself scattered by thermally excited domain walls $[39,42]$. This increases the effective localization of both continuum and 


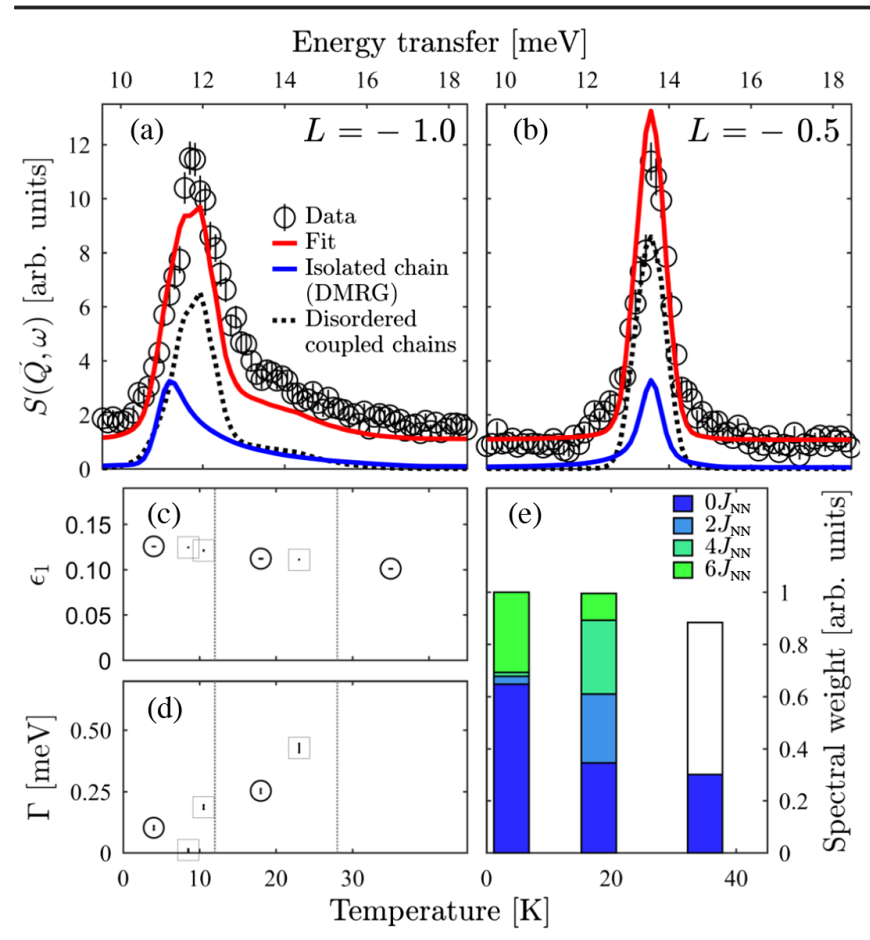

FIG. 4. (a),(b) Integrated scattered intensities at $35 \mathrm{~K}$ following the conventions of Figs. 2(c) and 2(d). Red lines show fits combining DMRG for the isolated chain (solid blue) and Matsubara-framework modeling of coupled chains with static thermal disorder (dashed black lines). (c) Effective $\epsilon_{1}(T)$, with circles (squares) from fits to high- (low-) statistics datasets. (d) Effective Lorentzian width $\Gamma(T)$. (e) Integrated intensity associated with each staggered field at 4 and $18 \mathrm{~K}$, taken from DMRG fits; at $35 \mathrm{~K}$ we distinguish the intensity (blue) associated with the isolated-chain response from that (white) due to chains in thermally disordered effective fields.

bound modes, as well as reducing their lifetimes, which changes the line shapes into Voigt functions with a constant Gaussian width of $0.32 \mathrm{meV}$ (FWHM instrument resolution) and a temperature-dependent Lorentzian width $\Gamma(T)$, which we take to be $\vec{Q}$ independent. In the Matsubara framework we model the band flattening due to the increased localization by a temperature-driven decrease of $\epsilon_{1}$ in Eq. (1) [44], taking $\epsilon_{2}$ as a constant. Below, we compute the spectral functions at all temperatures from DMRG calculations based only on the low- $T$ parameters, and use the effective $\Gamma(T)$ and $\epsilon_{1}(T)$ as an aid to physical interpretation.

A fully quantitative account of continuum and boundstate energies, of line shapes, and of the overall scattered intensities [color contours in Figs. 2(b), 3(b), and 1(b)] is obtained with the parameter values $J_{1}=5.89(1) \mathrm{meV}$, $J_{2}=-0.518(1) \mathrm{meV}, J_{\mathrm{NN}}=0.129(1) \mathrm{meV}$, and $\epsilon_{2}=$ $0.605(1)$, valid at all temperatures, and with $\epsilon_{1}(T)=$ $0.126(1)$ at $4 \mathrm{~K}, 0.112(1)$ at $18 \mathrm{~K}$, and $0.101(2)$ at $35 \mathrm{~K}$ [Fig. 4(c)]; $\Gamma(T)=0.102(8) \mathrm{meV}$ at $4 \mathrm{~K}$ and $0.25(1) \mathrm{meV}$ at $18 \mathrm{~K}$ [Fig. 4(d)], and is not defined at $35 \mathrm{~K}$ (below).
Figure 4(e) shows the optimized weights associated with chains in different effective fields at each temperature.

The separation of isolated-chain from staggered-field physics is clearest at $4 \mathrm{~K}$ [Fig. 2(a)]. The former is responsible for the $11 \mathrm{meV}$ peak in Fig. 2(c), whose continuum tail extends to $17 \mathrm{meV}$, and the latter, with $h=6 J_{\mathrm{NN}}$, for the sharp mode at $12.5 \mathrm{meV}$. The separation of these two primary contributions is emphasized by the small $\Gamma$ and the near absence of the intermediate staggered fields. We fit the relative weights of the staggered fields as 67(3), 3(3), 0(3), and 31(3)\% for $h$ from 0 to $6 J_{\mathrm{NN}}$, and thus the 0 and $6 J_{\mathrm{NN}}$ components are rather close to the ideal $2: 1$ ratio of the FI phase.

The most marked difference at $18 \mathrm{~K}$ [Fig. 3] is the strong shift toward contributions from the staggered fields $2 J_{\mathrm{NN}}$ and $4 J_{\mathrm{NN}}$, whose spectra overlap due both to their proximity in energy and to the increased $\Gamma$. The populations of the four staggered fields are 39(5), 35(5), 27(5), and $0(5) \%$, which lie close to our optimal CHB Monte Carlo results of $39,35,20$, and $6 \%$.

Finally, at $35 \mathrm{~K}$ one may expect interchain correlations to be lost and the scattering to be dominated by isolated-chain physics, as suggested by Fig. 1(c). However, in Fig. 4(a) it is clear that the isolated chain cannot provide an acceptable fit. To model the effects of thermal disorder, both in localizing the in-chain dynamics and in randomizing the chain environment, we have conducted Monte Carlo simulations of different static domain-wall distributions within the Matsubara framework, as described in Sec. S5 of the SM [34]. Qualitatively, we find a broadening dictated by $2 J_{\mathrm{NN}}$ that becomes independent of the chain-length distribution, and a shift of weight toward the band center that again is a localization effect reproduced using $\epsilon_{1}$. Quantitatively, however, a definitive fit of the $35 \mathrm{~K}$ data would require a systematic account of domain-wall dynamics.

For such a microscopic analysis of combined quantum and thermal fluctuations in the Ising chain, we have performed time-dependent DMRG calculations [45,46] to obtain the finite-temperature spectral functions $S(\vec{Q}, \omega, T)$, as described in Sec. S3 of the SM [34]. The spatial and temporal evolution of a spin-flip excitation was computed for 512-site chains and a linear prediction method [47] used to access long effective times despite rapid entanglement growth at the higher temperatures. At $4 \mathrm{~K}$ we obtain the optimized DMRG parameters $J_{1}=5.86 \mathrm{meV}, \quad J_{2}=-0.576 \mathrm{meV}, \quad J_{\mathrm{NN}}=0.128 \mathrm{meV}$, $\epsilon_{1}=0.126$, and $\epsilon_{2}=0.559$. By including the different staggered fields and the instrumental resolution, we obtain the spectrum shown in Fig. 2(b), where the DMRG and extended Matsubara results are indistinguishable. For a quantitative visualization of the accuracy with which the experimental peaks and line shapes are reproduced, the DMRG results for all staggered fields are also shown as the solid blue-to-green lines in Figs. 2(c) and 2(d), and in detail in Sec. S4 of the SM [34]. 
DMRG captures all of the thermal broadening effects arising from domain-wall scattering in a single chain. As Fig. 3 makes clear, this provides an excellent account of the $18 \mathrm{~K}$ spectrum in all its details, although the fitted chain population distribution of $35,27,28$, and $10 \%$ is somewhat different from the less-constrained Matsubara fit (Sec. S4 of the SM [34]). At $35 \mathrm{~K}$, where the approximation of chains in a well-ordered staggered field is no longer appropriate, we use our DMRG results for the scattered intensity $I_{0}(\omega)$ of the isolated chain to reproduce the contributions of chains decoupled from their neighbors by thermal fluctuations. In combination with the contribution of chains coupled by the random fields of a thermal distribution of static domain walls, which as above we model in the Matsubara framework, our optimal fit to the measured intensity [Figs. 4(a) and 4(b)] reveals that approximately $63(5) \%$ of the spectral weight, marked in white in Fig. 4(e), is contributed by chain segments in nonvanishing effective fields (Sec. S5 of the SM [34]). Thus we quantify the extent to which interchain correlations remain important at $T>T_{N 1}$ [28], i.e., close to but above the regime of finite long-range order.

We comment that higher modes of the Zeeman ladder are observed in the spectrum between 15 and $18 \mathrm{meV}$. These are rather sharp at $4 \mathrm{~K}$ [Figs. 2(a) and 2(c)] but broad and weak at $18 \mathrm{~K}$ [Fig. 3(a)]. In our Matsubara and DMRG results [Fig. 2(b)], these modes are present with approximately the measured position, but their dispersion and intensity cannot be fitted with the same quantitative accuracy as the other spectral features. This suggests that higher-energy corrections [25] or an RPA-type extension of the Matsubara formalism may be required.

To summarize, we have studied both the continuum and the bound-state excitations of the quasi-1D AFM Ising chain in a single material, $\mathrm{RbCoCl}_{3}$. By using temperature to control the type of $3 \mathrm{D}$ magnetic order, we measure the dynamical response in different effective magnetic fields. We model the continuum of the isolated chain, the Zeeman ladders in all staggered fields, and the broad response in thermally randomized fields by an analytical domain-wall formalism and by DMRG, to obtain a quantitative description of the spectrum in each of the temperature regimes investigated. Our results constitute a frontier in exploring the finite-temperature response of quantum spin systems and highlight the need for systematic theoretical methods to treat this problem in $3 \mathrm{D}$.

We are grateful to R. Coldea and F. Essler for helpful discussions. We thank the neutron scattering facilities ISIS (Rutherford Appleton Laboratory, UK), SINQ (Paul Scherrer Institute, Switzerland), and the Institut LaueLangevin (France). This research was supported by the UK Engineering and Physical Sciences Research Council (EPSRC) under Grant No. EP/N027671/1, by the European Research Council (ERC) under the EU Horizon 2020 research and innovation program Grants No. 681654
(HyperQC) and No. 742102 (QUENOCOBA), by the Bavarian Elite Network ExQM, and by the Swiss National Science Foundation (SNF) under Grants No. 200020-132877 and No. 200020-150257.

[1] T. Giamarchi, Quantum Physics in One Dimension (Oxford University Press, Oxford, 2004).

[2] E. Ising, Z. Phys. 31, 253 (1925).

[3] H. Yoshizawa, K. Hirakawa, S. K. Satija, and G. Shirane, Phys. Rev. B 23, 2298 (1981).

[4] S. E. Nagler, W. J. L. Buyers, R. L. Armstrong, and B. Briat, Phys. Rev. B 27, 1784 (1983).

[5] S. E. Nagler, W. J. L. Buyers, R. L. Armstrong, and B. Briat, Phys. Rev. B 28, 3873 (1983).

[6] R. Coldea, D. A. Tennant, E. M. Wheeler, E. Wawrzynska, D. Prabhakaran, M. Telling, K. Habicht, P. Smeibidl, and K. Kiefer, Science 327, 177 (2010).

[7] S. Sachdev, Phys. World 12, 33 (1999).

[8] H. M. Rønnow, R. Parthasarathy, J. Jensen, G. Aeppli, T. F. Rosenbaum, and D. F. McMorrow, Science 308, 389 (2005).

[9] C. Kraemer, N. Nikseresht, J. O. Piatek, N. Tsyrulin, B. D. Piazza, K. Kiefer, B. Klemke, T. F. Rosenbaum, G. Aeppli, C. Gannarelli, K. Prokes, A. Podlesnyak, T. Strässle, L. Keller, O. Zaharko, K. W. Krämer, and H. M. Rønnow, Science 336, 1416 (2012).

[10] Q. Faure, S. Takayoshi, S. Petit, V. Simonet, S. Raymond, L.-P. Regnault, M. Boehm, J. S. White, M. Månsson, Ch. Rüegg, P. Lejay, B. Canals, S. C. Furuya, T. Giamarchi, and B. Grenier, Nat. Phys. 14, 716 (2018).

[11] C. M. Morris, R. Valdés Aguilar, A. Ghosh, S. M. Koohpayeh, J. Krizan, R. J. Cava, O. Tchernyshyov, T. M. McQueen, and N. P. Armitage, Phys. Rev. Lett. 112, 137403 (2014).

[12] N. J. Robinson, F. H. L. Essler, I. Cabrera, and R. Coldea, Phys. Rev. B 90, 174406 (2014).

[13] I. Cabrera, J. D. Thompson, R. Coldea, D. Prabhakaran, R. I. Bewley, T. Guidi, J. A. Rodriguez-Rivera, and C. Stock, Phys. Rev. B 90, 014418 (2014).

[14] S. Kimura, H. Yashiro, K. Okunishi, M. Hagiwara, Z. He, K. Kindo, T. Taniyama, and M. Itoh, Phys. Rev. Lett. 99, 087602 (2007).

[15] S. Kimura, K. Okunishi, M. Hagiwara, K. Kindo, Z. He, T. Taniyama, M. Itoh, K. Koyama, and K. Watanabe, J. Phys. Soc. Jpn. 82, 033706 (2013).

[16] A. K. Bera, B. Lake, W.-D. Stein, and S. Zander, Phys. Rev. B 89, 094402 (2014).

[17] Z. Wang, M. Schmidt, A. K. Bera, A. T. M. N. Islam, B. Lake, A. Loidl, and J. Deisenhofer, Phys. Rev. B 91, 140404 (R) (2015).

[18] B. Grenier, S. Petit, V. Simonet, E. Canévet, L.-P. Regnault, S. Raymond, B. Canals, C. Berthier, and P. Lejay, Phys. Rev. Lett. 114, 017201 (2015).

[19] A. K. Bera, B. Lake, F. H. L. Essler, L. Vanderstraeten, C. Hubig, U. Schollwöck, A. T. M. N. Islam, A. Schneidewind, and D. L. Quintero-Castro, Phys. Rev. B 96, 054423 (2017).

[20] Z. Wang, J. Wu, S. Xu, W. Yang, C. Wu, A. K. Bera, A. T. M. Nazmul Islam, B. Lake, D. Kamenskyi, P. Gogoi, H. Engelkamp, N. Wang, J. Deisenhofer, and A. Loidl, Phys. Rev. B 94, 125130 (2016). 
[21] M. Matsuda, H. Onishi, A. Okutani, J. Ma, H. Agrawal, T. Hong, D. M. Pajerowski, J. R. D. Copley, K. Okunishi, M. Mori, S. Kimura, and M. Hagiwara, Phys. Rev. B 96, 024439 (2017).

[22] Z. Wang, J. Wu, W. Yang, A. K. Bera, D. Kamenskyi, A. T. M. N. Islam, S. Xu, J. M. Law, B. Lake, C. Wu, and A. Loidl, Nature (London) 554, 219 (2018).

[23] W. Lehmann, W. Breitling, and R. Weber, J. Phys. C 14, 4655 (1981).

[24] F. Matsubara, S. Inawashiro, and H. Ohhara, J. Phys. Condens. Matter 3, 1815 (1991).

[25] J. P. Goff, D. A. Tennant, and S. E. Nagler, Phys. Rev. B 52, 15992 (1995).

[26] D. J. Lockwood, I. W. Johnstone, H. J. Labbe, and B. Briat, J. Phys. C 16, 6451 (1983).

[27] A. Oosawa, Y. Nishiwaki, T. Kato, and K. Kakurai, J. Phys. Soc. Jpn. 75, 015002 (2006).

[28] N. P. Hänni, D. Sheptyakov, U. Stuhr, L. Keller, M. Medarde, A. Cervellino, L.-P. Regnault, E. Hirtenlechner, M. Mena, Ch. Rüegg, and K. W. Krämer (unpublished).

[29] N. Ishimura and H. Shiba, Prog. Theor. Phys. 63, 743 (1980).

[30] H. Shiba, Prog. Theor. Phys. 64, 466 (1980).

[31] R. I. Bewley, J. W. Taylor, and S. M. Bennington, Nucl. Instrum. Methods Phys. Res., Sect. A 637, 128 (2011).

[32] Available at http://www.mantidproject.org.

[33] R. A. Ewings, A. Buts, M. D. Le, J. van Duijn, I. Bustinduy, and T. G. Perring, Nucl. Instrum. Methods Phys. Res., Sect. A 834, 132 (2016).
[34] See Supplemental Material at http://link.aps.org/ supplemental/10.1103/PhysRevLett.124.257201, which includes Refs. [35-37], for details.

[35] F. Verstraete, J. J. Garcia-Ripoll, and J. I. Cirac, Phys. Rev. Lett. 93, 207204 (2004).

[36] J. Haegeman, C. Lubich, I. Oseledets, B. Vandereycken, and F. Verstraete, Phys. Rev. B 94, 165116 (2016).

[37] T. Barthel, New J. Phys. 15, 073010 (2013).

[38] F. Matsubara, A. Sato, O. Koseki, and T. Shirakura, Phys. Rev. Lett. 78, 3237 (1997).

[39] O. Koseki and F. Matsubara, J. Phys. Soc. Jpn. 66, 322 (1997).

[40] O. Koseki and F. Matsubara, J. Phys. Soc. Jpn. 69, 1202 (2000).

[41] M. Mekata, J. Phys. Soc. Jpn. 42, 76 (1977).

[42] N. Todoroki and S. Miyashita, J. Phys. Soc. Jpn. 73, 412 (2004).

[43] H. Shiba, Y. Ueda, K. Okunishi, S. Kimura, and K. Kindo, J. Phys. Soc. Jpn. 72, 2326 (2003).

[44] A. J. A. James, W. D. Goetze, and F. H. L. Essler, Phys. Rev. B 79, 214408 (2009).

[45] U. Schollwöck, Ann. Phys. (Amsterdam) 326, 96 (2011).

[46] C. Hubig, I. P. McCulloch, and U. Schollwöck, Phys. Rev. B 95, 035129 (2017).

[47] T. Barthel, U. Schollwöck, and S. R. White, Phys. Rev. B 79, 245101 (2009). 